\title{
Infrared Observations of the Crab Nebula
}

\author{
Yves A. Gallant \\ Dublin Institute for Advanced Studies, Dublin, Ireland, and \\ Astronomical Institute, Utrecht University, Netherlands
}

Richard J. Tuffs

Max-Planck-Institut für Kernphysik, Heidelberg, Germany

\begin{abstract}
We obtained narrow-band continuum images of the Crab Nebula at $16,6.4$ and $2.2 \mu \mathrm{m}$. From these we derive spectral index maps, extending the optical work of Véron-Cetty \& Woltjer (1993). In the central parts of the Nebula the IR spectral index is close to the radio one, indicating the region where the radio-emitting electrons are accelerated.
\end{abstract}

\section{Introduction and Motivation}

The synchrotron spectrum of the Crab Nebula extends from radio to gammarays, with increasingly steep power-law spectral indices $\alpha$, where $F_{\nu} \propto \nu^{-\alpha}$. The spectral index is a remarkably uniform $\alpha_{r} \approx 0.3$ in the radio, but $\alpha_{X} \approx 1.15$ in $\mathrm{X}$-rays. As synchrotron aging implies a steepening of $\alpha$ by only 0.5 , it is unclear how the electron populations responsible for the radio and $\mathrm{X}$-ray emission are related. The model of Kennel and Coroniti (1984) accounts fairly well for the optical to X-ray emission, but leaves the "radio electrons" unexplained.

Véron-Cetty \& Woltjer (1993) mapped the spectral index of the Nebula in the optical, and found a harder spectral index region in the center with morphology similar to that in X-rays, and gradual steepening of the spectrum towards the edges of the Nebula. Precise spectral index determinations in the optical are made difficult by uncertainties in the amount of interstellar reddening to the Crab, however. Our infrared observations were motivated in part by the fact that the extinction is much less in this band, as well as by the aim of better constraining the transition between the radio and optical spectral indices.

\section{Observations and Analysis}

We performed raster observations with ISOCAM on board ESA's ISO satellite, using two fixed CVF narrow bandpasses around 16.0 and $6.4 \mu \mathrm{m}$ chosen to minimise contributions from known lines. We also obtained an image in a narrow-band continuum filter around $2.2 \mu \mathrm{m}$ at the Calar Alto Observatory. The angular resolution was limited by the ISOCAM pixel size, fixed at $6^{\prime \prime}$ but sampled with half-pixel steps. Figure 1 (left) shows a preliminary spectral index map between 16 and $2.2 \mu \mathrm{m}$, obtained after correction for interstellar reddening. 

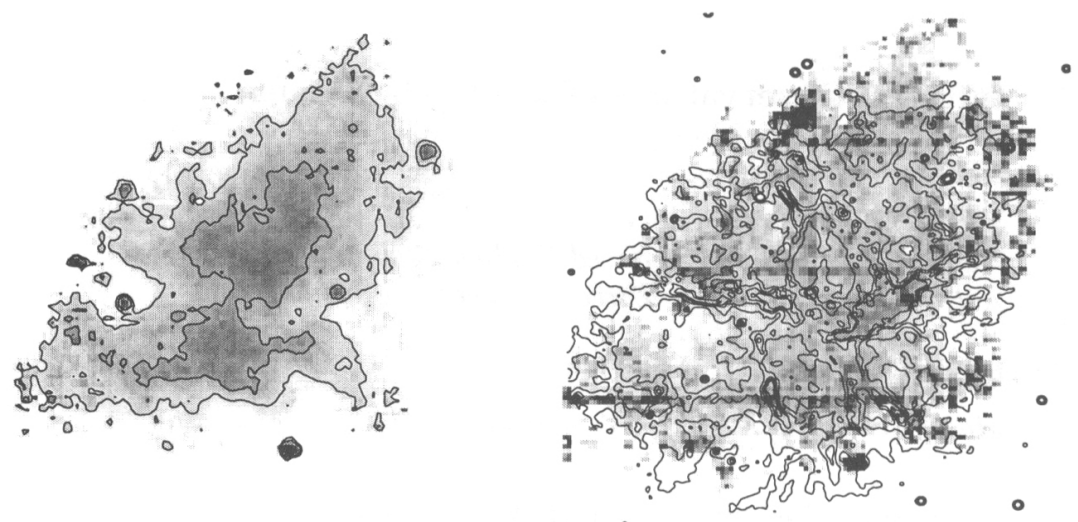

Figure 1. Left panel: Spectral index $\alpha$ between 16.0 and $2.2 \mu \mathrm{m}$; contours are at 0.4 and 0.6 . Right panel: Spectral curvature, as measured at $6.4 \mu \mathrm{m}$; contours of an optical [OIII] image have been superimposed.

The shrinking of the Nebula with increasing frequency is apparent in the steepening of the spectral index towards the edges. The flattest spectral index values are found in the central regions, where $\alpha$ reaches about 0.3 , the same value as in the radio. Figure 1 (right) shows spectral curvature, measured as the ratio of interpolated to actual fluxes at $6.4 \mu \mathrm{m}$. The spectrum is convex, i.e. steepening with increasing frequency, near the edges, but concave in regions which appear concident with line-emitting filaments. This suggests a $16 \mu \mathrm{m}$ continuum excess in the filaments, perhaps due to a steeper synchrotron component or to dust.

\section{Discussion}

The spectral index in the central part of the Nebula being close to the radio value suggests that we are seeing the continuation of the same electron population, and that the gradual steepening towards the edges of the Nebula is due to synchrotron losses. While weaker magnetic fields in the central Nebula are a possibility, the more obvious interpretation is that the "radio electrons" are accelerated in this harder spectral index region, and diffuse or are advected into the outer Nebula. The connection, if any, between this "radio electron" population seen in the infrared and the "X-ray electron" population seen in the optical still remains to be elucidated.

Acknowledgments. This collaboration was supported by the European Union under the TMR programme, contract number FMRX-CT98-0168.

\section{References}

Kennel, C. F., \& Coroniti, F. V. 1984, ApJ, 283, 710

Véron-Cetty, M. P., \& Woltjer, L. 1993, A\& $4,270,370$ 